\title{
Macroparasites of epipelagic and eurybathic fishes in the north-western Pacific
}

\author{
I.I. Gordeev ${ }^{1,2}$, S.G. Sokolov ${ }^{3}$ \\ ${ }^{1}$ Lomonosov Moscow State University, Leninskiye Gory 1/12, Moscow 119234 Russia. E-mail: \\ gordeev_ilya@bk.ru \\ ${ }^{2}$ Russian Federal Research Institute of Fisheries and Oceanography, Verkhn. Krasnoselskaya Str. \\ 17, Moscow 107140 Russia. \\ ${ }^{3}$ A.N. Severtsov Institute of Ecology and Evolution of the RAS, Leninskiy pr. 33, Moscow 119071 \\ Russia.E-mail:sokolovsg@mail.ru
}

ABSTRACT: A total of 25 parasitic species were found to infect 23 species of teleost and two species of cartilaginous fish in the north-western Pacific. Known generalists, anisakid and raphidascaridid juveniles (Nematoda), plerocercoids of Nybelinia surmenicola (Cestoda), and acanthocephalan Echinorhynchus gadi (Acanthocephala) were prevalent; however, plerocercoids of Pelichnibothrium speciosum were the most common parasites of epipelagic and eurybathic fish, infecting 16 out of 23 examined teleost species. Digenean infection of eurybathic species Aptocyclus ventricosus (Cyclopteridae), Zaprora silenus (Zaproridae), Leuroglossus schmidti (Bathylagidae), and Icichthys lockingtoni (Centrolophidae) differ from other teleost fish in their parasite fauna. The present study is the first report of macroparasites from Magnisudis atlantica (Paralepididae) and I. lockingtoni. In addition, this is the first data on the infection of Gasterosteus aculeatus (Gasterosteidae) far into the open ocean. Macroparasite infection of fish in the epipelagic layer of the northwestern Pacific Ocean is characterised by high infection rates and low species diversity of parasites.

How to cite this article: Gordeev I.I., Sokolov S.G. 2020. Macroparasites of epipelagic and eurybathic fishes in the north-western Pacific // Invert. Zool. Vol.17. No.2. P.118-132. doi: 10.15298/invertzool.17.2.02

KEY WORDS: helminths, Pacific Ocean, fish parasites, marine biology, parasitology.

\section{Макропаразиты эпипелагических и эврибатных рыб северо-западной части Тихого океана}

\section{И.И. Гордеев ${ }^{1,2}$, С.Г. Соколов ${ }^{3}$}

\footnotetext{
${ }^{1}$ Московский государственный университет им. М.В. Ломоносова, Ленинские горы, д. 1/12, Москва 119234 Россия. E-mail: gordeevilya@bk.ru

${ }^{2}$ Всероссийский научно-исследовательский институт рыбного хозяйства и океанографии, ул. Верхняя Красносельская 17, Москва 107140 Россия.

${ }^{3}$ Институт проблем экологии и эволючии им. А.Н. Северцова РАН, Ленинский пр. 33, Москва 119071 Россия. E-mail: sokolovsg@mail.ru
}

РЕЗЮМЕ: В ходе паразитологического исследования рыб в северо-западной части Тихого океана было обследовано 23 вида костистых и 2 вида хрящевых рыб, в которых обнаружено 25 видов паразитов. Известные генералисты, такие как личинки 
анизакидных и рафидаскаридидных нематод, плероцеркоиды Nybelinia surmenicola и скребень Echinorhynchus gadi встречались часто, однако, наиболее массовыми были плероцеркоиды Pelichnibothrium speciosum, найденные в 16 из 23 обследованных видов костистых рыб. Трематоды были обнаружены в эврибатных видах рыбAptocyclus ventricosus (Cyclopteridae), Zaprora silenus (Zaproridae), Leuroglossus schmidti (Bathylagidae) и Icichthys lockingtoni (Centrolophidae), что заметно отличало их от других обследованных видов. Макропаразиты рыб Magnisudis atlantica (Paralepididae) и I. lockingtoni исследованы впервые, также впервые получены данные о зараженности трехиглой колюшки Gasterosteus aculeatus (Gasterosteidae) в открытых водах Тихого океана на значительном удалении от берега. В целом, инвазия рыб макропаразитами в эпипелагиали северо-западной части Тихого океана характеризуется значительной интенсивностью инвазии, однако бедным видовым составом.

Как цитировать эту статью: Gordeev I.I., Sokolov S.G. 2020. Macroparasites of epipelagic and eurybathic fishes in the north-western Pacific // Invert. Zool. Vol.17. No.1. P.118-132. doi: 10.15298/invertzool.17.2.02

КЛЮЧЕВЫЕ СЛОВА: гельминты, Тихий океан, паразиты рыб, морская среда, паразитология.

\section{Introduction}

The study of the open ocean is a hard task, due to its depth, size, and distance from the centres of civilisation. The Pacific Ocean, occupying half the globe, can compete with the Southern Ocean in its degree of unexploredness (Rhode, 2005). In the polar and circumpolar, the most productive regions of the Pacific Ocean, there is a large number of concentrated fisheries, aimed at such valuable fish species as salmon, Oncorhynchus spp. (Salmonidae) (Gordeev, Klovach, 2019), walleye pollock, Gadus chalcogrammus Pallas, 1814 (Gadidae), Pacific herring, Clupea pallasii Valenciennes, 1847 (Clupeidae), Pacific saury, Cololabis saira (Brevoort, 1856) (Scomberesocidae), chub mackerel, Scomber japonicus (Houttuyn, 1782) (Scombridae), and halibuts, Hippoglossus spp. and Atheresthes spp. (Pleuronectidae).

The main reason to study the biodiversity and biology of parasites is their role in ecosystem functioning. There is increasing evidence that parasite-mediated effects could be significant. They shape host population dynamics, alter interspecific competition, influence energy flow, and appear to be important drivers of biodiversity (Hudson et al., 2006). Also, para- sites appear to have an important role in influencing organisation within communities via reducing host fitness, modifying competitive and trophic interactions among species, altering the behaviour of the host and in other ways (Hudson et al., 2006). The role of parasites and their hosts in marine invasions, when invaders directly or indirectly affect parasite and host populations and communities, is an actively studied area of fish parasitology (Goedknegt et al., 2016). Undoubtedly, data on the infection of wild fish populations are the primary data to elucidate the fundamental role of parasites in marine ecosystem functioning. Moreover, parasites are of undoubted importance for the economy, since they not only cause significant damage to a fishery product's marketable condition, but also sometimes make it unsafe to use it for food (e.g. Quiazon, 2015). While the knowledge of parasites is sufficient for commercially important fish species, the great majority of other fish have never been investigated in this respect (Klimpel et al., 2009). There are many species that inhabit the same water horizon $(0-$ $50 \mathrm{~m}$ ) as salmon during their period in the marine environment (Gordeev et al., 2018), and parasitological examination of these species is interesting in terms of broadening our understanding of helminth infection in the open ocean. 
In the present paper, we summarise the results of the parasitological examination of 25 species of teleost and cartilaginous, epipelagic and eurybathic fishes in the north-western Pacific Ocean performed aboard the $R V$ Professor Kaganovsky in June-July 2018 (Gordeev et al., 2018).

\section{Material and Methods}

All specimens were caught between 31 May and 9 July 2018 by the research vessel Professor Kaganovsky during a survey of Pacific salmon (stock assessment), using midwater net trawl RT/TM 80/396, with a $10 \mathrm{~mm}$ mesh insert in the

Table 1. Length, weight and locations of catches of fish examined during the parasitological survey on the $R V$ Professor Kaganovsky.

Таблица 1. Длина, вес и координаты вылова особей, исследованных в ходе паразитологического исследования на НИС «Профессор Кагановский».

\begin{tabular}{|c|c|c|}
\hline $\begin{array}{l}\text { Species / total length range, } \\
\text { weight range }\end{array}$ & $\begin{array}{l}\text { Total no. } \\
\text { examined }\end{array}$ & Localities (no. examined) \\
\hline 1 & 2 & 3 \\
\hline \multicolumn{3}{|l|}{ Salmoniformes } \\
\hline $\begin{array}{l}\text { Oncorhynchus gorbuscha } \\
\text { (Walbaum, 1792)/42.0-46.2 } \\
\mathrm{cm}, 717-959 \mathrm{~g}\end{array}$ & 15 & $\begin{array}{l}45^{\circ} 06^{\prime} \mathrm{N} \& 149^{\circ} 39^{\prime} \mathrm{E}(1) ; 49^{\circ} 59^{\prime} \mathrm{N} \& 156^{\circ} 16^{\prime} \mathrm{E} \\
(2) ; 50^{\circ} 45^{\prime} \mathrm{N} \& 159^{\circ} 57^{\prime} \mathrm{E}(1) ; \\
46^{\circ} 09^{\prime} \mathrm{N} \& 155^{\circ} 49^{\prime} \mathrm{E}(1) ; 43^{\circ} 58^{\prime} \mathrm{N} \& 152^{\circ} 16^{\prime} \mathrm{E} \\
(1) ; 43^{\circ} 14^{\prime} \mathrm{N} \& 148^{\circ} 28^{\prime} \mathrm{E}(2) ; \\
46^{\circ} 00^{\prime} \mathrm{N} \& 157^{\circ} 46^{\prime} \mathrm{E}(1) ; 46^{\circ} 55^{\prime} \mathrm{N} \& 158^{\circ} 22^{\prime} \mathrm{E} \\
(2) ; 49^{\circ} 06^{\prime} \mathrm{N} \& 161^{\circ} 58^{\prime} \mathrm{E}(4)\end{array}$ \\
\hline $\begin{array}{l}\text { Oncorhynchus keta } \\
\text { (Walbaum, 1792)/42.9-56.0 } \\
\mathrm{cm}, 902-1966 \mathrm{~g}\end{array}$ & 15 & $\begin{array}{l}49^{\circ} 59^{\prime} 2 \& 156^{\circ} 16^{\prime} \mathrm{E}(3) ; 49^{\circ} 24^{\prime} \mathrm{N} \& 159^{\circ} 56^{\prime} \mathrm{E} \\
(2) ; 46^{\circ} 27^{\prime} \mathrm{N} \& 161^{\circ} 53^{\prime} \mathrm{E}(5) ; \\
43^{\circ} 57^{\prime} \mathrm{N} \& 149^{\circ} 31^{\prime} \mathrm{E}(5) ;\end{array}$ \\
\hline $\begin{array}{l}\text { Oncorhynchus kisutch } \\
\text { (Walbaum, 1792)/44.6-53.6 } \\
\mathrm{cm}, 1072-1908 \mathrm{~g}\end{array}$ & 3 & $46^{\circ} 27^{\prime} \mathrm{N} \& 166^{\circ} 21^{\prime} \mathrm{E}(3)$ \\
\hline \multicolumn{3}{|l|}{ Perciformes } \\
\hline $\begin{array}{l}\text { Brama japonica Hilgendorf, } \\
1878 / 40.8-45.2 \mathrm{~cm}, 1217- \\
1833 \mathrm{~g}\end{array}$ & 13 & $42^{\circ} 41^{\prime} \mathrm{N} \& 154^{\circ} 38^{\prime} \mathrm{E}(13)$ \\
\hline $\begin{array}{l}\text { Hyperoglyphe japonica } \\
\text { (Döderlein, 1884) / 21.0-38.7 } \\
\mathrm{cm}, 267-469 \mathrm{~g}\end{array}$ & 2 & $\begin{array}{l}42^{\circ} 18^{\prime} \mathrm{N} \& 146^{\circ} 31^{\prime} \mathrm{E}(1) ; 41^{\circ} 48^{\prime} \mathrm{N} \& 150^{\circ} 41^{\prime} \mathrm{E} \\
\text { (1) }\end{array}$ \\
\hline $\begin{array}{l}\text { Icichthys lockingtoni Jordan et } \\
\text { Gilbert, } 1880 / 37.0 \mathrm{~cm}, 398 \mathrm{~g}\end{array}$ & 2 & $\begin{array}{l}41^{\circ} 50^{\prime} \mathrm{N} \& 148^{\circ} 45^{\prime} \mathrm{E}(1) ; 43^{\circ} 26^{\prime} \mathrm{N} \& 155^{\circ} 56^{\prime} \mathrm{E} \\
\text { (1) }\end{array}$ \\
\hline $\begin{array}{l}\text { Zaprora silenus Jordan, } 1896 \text { / } \\
23.0-31.0 \mathrm{~cm}, 90-471 \mathrm{~g}\end{array}$ & 6 & $\begin{array}{l}47^{\circ} 31^{\prime} \mathrm{N} \& 155^{\circ} 31^{\prime} \mathrm{E}(1) ; 44^{\circ} 41^{\prime} \mathrm{N} \& 153^{\circ} 48^{\prime} \mathrm{E} \\
(1) ; 46^{\circ} 00^{\prime} \mathrm{N} \& 157^{\circ} 46^{\prime} \mathrm{E}(1) ; \\
49^{\circ} 02^{\prime} \mathrm{N} \& 163^{\circ} 27^{\prime} \mathrm{E}(1) ; 49^{\circ} 58^{\prime} \mathrm{N} \& 167^{\circ} 45^{\prime} \mathrm{E} \\
\text { (2); }\end{array}$ \\
\hline \multicolumn{3}{|l|}{ Aulopiformes } \\
\hline $\begin{array}{l}\text { Lestidiops ringens (Jordan et } \\
\text { Gilbert, 1880) } / 17.0-21.0 \mathrm{~cm} \text {, } \\
8-12 \mathrm{~g}\end{array}$ & 30 & $48^{\circ} 11^{\prime} \mathrm{N} \& 166^{\circ} 10^{\prime} \mathrm{E}(30)$ \\
\hline $\begin{array}{l}\text { Magnisudis atlantica (Krøyer, } \\
1868) / 38.0 \mathrm{~cm}, 185 \mathrm{~g}\end{array}$ & 1 & $45^{\circ} 09^{\prime} \mathrm{N} \& 152^{\circ} 18^{\prime} \mathrm{E}(1)$ \\
\hline $\begin{array}{l}\text { Alepisaurus ferox Lowe, } 1833 \\
/ 100.2-133.0 \mathrm{~cm}, 1057-4016 \\
\mathrm{~g}\end{array}$ & 4 & $\begin{array}{l}50^{\circ} 06 \mathrm{~N} \& 158^{\circ} 50^{\prime} \mathrm{E}(1) ; 46^{\circ} 58^{\prime} \mathrm{N} \& 156^{\circ} 56^{\prime} \mathrm{E} \\
(1) ; 46^{\circ} 37^{\prime} \mathrm{N} \& 159^{\circ} 56^{\prime} \mathrm{E}(1) ; \\
50^{\circ} 33^{\prime} \mathrm{N} \& 165^{\circ} 30^{\prime} \mathrm{E}(1)\end{array}$ \\
\hline
\end{tabular}


Table 1 (continued). Таблица 1 (продолжение).

\begin{tabular}{|c|c|c|}
\hline 1 & 2 & 3 \\
\hline \multicolumn{3}{|l|}{ Aulopiformes } \\
\hline $\begin{array}{l}\text { Scopelosaurus harryi (Mead, } \\
1953) / 11.5-22.0 \mathrm{~cm}, 5-49 \mathrm{~g}\end{array}$ & 2 & $\begin{array}{l}42^{\circ} 55^{\prime} \mathrm{N} \& 153^{\circ} 03^{\prime} \mathrm{E}(1) ; 42^{\circ} 41^{\prime} \mathrm{N} \& 154^{\circ} 38^{\prime} \mathrm{E} \\
\text { (1) }\end{array}$ \\
\hline $\begin{array}{l}\text { Anotopterus nikparini } \\
\text { Kukuev, } 1998 / 43.0-114.0 \\
\mathrm{~cm}, 62-1778 \mathrm{~g}\end{array}$ & 9 & $\begin{array}{l}50^{\circ} 06^{\prime} \mathrm{N} \& 158^{\circ} 50^{\prime} \mathrm{E}(1) ; 47^{\circ} 48^{\prime} \mathrm{N} \& 157^{\circ} 56^{\prime} \mathrm{E} \\
(1) ; 46^{\circ} 58^{\prime} \mathrm{N} \& 156^{\circ} 56^{\prime} \mathrm{E}(1) ; \\
50^{\circ} 45^{\prime} \mathrm{N} \& 159^{\circ} 57^{\prime} \mathrm{E}(1) ; 46^{\circ} 55^{\prime} \mathrm{N} \& 158^{\circ} 22^{\prime} \mathrm{E} \\
(1) ; 49^{\circ} 02^{\prime} \mathrm{N} \& 163^{\circ} 27^{\prime} \mathrm{E}(1) ; \\
45^{\circ} 02^{\prime} \mathrm{N} \& 161^{\circ} 53^{\prime} \mathrm{E}(1) ; 45^{\circ} 09^{\prime} \mathrm{N} \& 152^{\circ} 18^{\prime} \mathrm{E} \\
(2)\end{array}$ \\
\hline \multicolumn{3}{|l|}{ Myctophiformes } \\
\hline $\begin{array}{l}\text { Diaphus theta Eigenmann et } \\
\text { Eigenmann, } 1890 / 4.1-7.5 \\
\mathrm{~cm}, 2.6-3.2 \mathrm{~g}\end{array}$ & 25 & $45^{\circ} 39^{\prime} \mathrm{N} \& 160^{\circ} 57^{\prime} \mathrm{E}(25)$ \\
\hline $\begin{array}{l}\text { Notoscopelus japonicus } \\
(\text { Tanaka, 1908)/11.5-14.0 } \\
\mathrm{cm}, 11.9-12.8 \mathrm{~g}\end{array}$ & 35 & $\begin{array}{l}43^{\circ} 14^{\prime} \mathrm{N} \& 148^{\circ} 28^{\prime} \mathrm{E}(10) ; \\
42^{\circ} 55^{\prime} \mathrm{N} \& 153^{\circ} 03^{\prime} \mathrm{E}(1) ; 42^{\circ} 55^{\prime} \mathrm{N} \& 153^{\circ} 03^{\prime} \mathrm{E} \\
(24) ;\end{array}$ \\
\hline $\begin{array}{l}\text { Stenobrachius leucopsarus } \\
\text { (Eigenmann et Eigenmann, } \\
1890) / 6.4-8.5 \mathrm{~cm}, 4.3-6.5 \mathrm{~g}\end{array}$ & 14 & $\begin{array}{l}44^{\circ} 41^{\prime} \mathrm{N} \& 153^{\circ} 48^{\prime} \mathrm{E} \\
42^{\circ} 55^{\prime} \mathrm{N} \& 153^{\circ} 03^{\prime} \mathrm{E}(2)\end{array}$ \\
\hline $\begin{array}{l}\text { Tarletonbeania crenularis } \\
\text { (Jordan et Gilbert, 1880)/ } 6.5 \\
\mathrm{~cm}, 4 \mathrm{~g}\end{array}$ & 1 & $42^{\circ} 41^{\prime} \mathrm{N} \& 154^{\circ} 38^{\prime} \mathrm{E}(1)$ \\
\hline \multicolumn{3}{|l|}{ Scorpaeniformes } \\
\hline $\begin{array}{l}\text { Aptocyclus ventricosus (Pallas, } \\
1769) / 3.0-29.0 \mathrm{~cm}, 1.12- \\
1385 \mathrm{~g}\end{array}$ & 15 & $\begin{array}{l}50^{\circ} 06 \mathrm{~N} \& 158^{\circ} 50^{\prime} \mathrm{E}(2) ; 47^{\circ} 31^{\prime} \mathrm{N} \& 155^{\circ} 31^{\prime} \mathrm{E} \\
(3) ; 45^{\circ} 56^{\prime} \mathrm{N} \& 153^{\circ} 20^{\prime} \mathrm{E}(1) ; \\
45^{\circ} 20^{\prime} \mathrm{N} \& 154^{\circ} 51^{\prime} \mathrm{E}(2) ; 45^{\circ} 09^{\prime} \mathrm{N} \& 152^{\circ} 18^{\prime} \mathrm{E} \\
(2) ; 44^{\circ} 58^{\prime} \mathrm{N} \& 158^{\circ} 12^{\prime} \mathrm{E}(2) ; \\
48^{\circ} 13^{\prime} \mathrm{N} \& 162^{\circ} 26^{\prime} \mathrm{E}(2) ; 47^{\circ} 20^{\prime} \mathrm{N} \& 167^{\circ} 07^{\prime} \mathrm{E} \\
(1)\end{array}$ \\
\hline \multicolumn{3}{|l|}{ Scombriformes } \\
\hline $\begin{array}{l}\text { Scomber japonicus Houttuyn, } \\
1782 / 19.9-40.2 \mathrm{~cm}, 77-529 \\
\mathrm{~g}\end{array}$ & 25 & $\begin{array}{l}43^{\circ} 58^{\prime} \mathrm{N} \& 152^{\circ} 16^{\prime} \mathrm{E}(5) ; 42^{\circ} 35^{\prime} \mathrm{N} \& 148^{\circ} 50^{\prime} \mathrm{E} \\
(5) ; 40^{\circ} 26^{\prime} \mathrm{N} \& 147^{\circ} 51^{\prime} \mathrm{E}(5) ; \\
43^{\circ} 26^{\prime} \mathrm{N} \& 155^{\circ} 56^{\prime} \mathrm{E}(10)\end{array}$ \\
\hline \multicolumn{3}{|l|}{ Beloniformes } \\
\hline $\begin{array}{l}\text { Cololabis saira (Brevoort, } \\
1856) / 26.8-39.5 \mathrm{~cm}, 11-154 \\
\mathrm{~g}\end{array}$ & 52 & $\begin{array}{l}43^{\circ} 26^{\prime} \mathrm{N} \& 155^{\circ} 56^{\prime} \mathrm{E}(1) ; 45^{\circ} 39^{\prime} \mathrm{N} \& 160^{\circ} 57^{\prime} \mathrm{E} \\
(27) ; 45^{\circ} 02^{\prime} \mathrm{N} \& 161^{\circ} 53^{\prime} \mathrm{E}(24)\end{array}$ \\
\hline \multicolumn{3}{|l|}{ Argentiniformes } \\
\hline $\begin{array}{l}\text { Leuroglossus schmidti Rass, } \\
1955 / 11.1-17.2 \mathrm{~cm}, 38.2- \\
43.1 \mathrm{~g}\end{array}$ & 16 & $50^{\circ} 06^{\prime} \mathrm{N} \& 158^{\circ} 50^{\prime} \mathrm{E}(16)$ \\
\hline \multicolumn{3}{|l|}{ Clupeiformes } \\
\hline $\begin{array}{l}\text { Sardinops sagax melanosticta } \\
\text { (Temminck et Schlegel, 1846) } \\
/ 16.2-22.6 \mathrm{~cm}, 39-114 \mathrm{~g}\end{array}$ & 10 & $40^{\circ} 26^{\prime} \mathrm{N} \& 147^{\circ} 51^{\prime} \mathrm{E}(10)$ \\
\hline
\end{tabular}


Table 1 (continued). Таблица 1 (продолжение).

\begin{tabular}{|c|c|c|}
\hline 1 & 2 & 3 \\
\hline \multicolumn{3}{|l|}{ Lampriformes } \\
\hline $\begin{array}{l}\text { Desmodema lorum Rosenblatt } \\
\text { et Butler, } 1977 / 113.0 \mathrm{~cm} \text {, } \\
640 \mathrm{~g}\end{array}$ & 1 & $41^{\circ} 29^{\prime} \mathrm{N} \& 147^{\circ} 16^{\prime} \mathrm{E}(1)$ \\
\hline \multicolumn{3}{|l|}{ Gasterosteiformes } \\
\hline $\begin{array}{l}\text { Gasterosteus aculeatus } \\
\text { Linnaeus, } 1758 / 4.5-8.7 \mathrm{~cm} \text {, } \\
2.16-4.17 \mathrm{~g}\end{array}$ & 214 & $\begin{array}{l}50^{\circ} 43^{\prime} \mathrm{N} \& 164^{\circ} 05^{\prime} \mathrm{E}(14) ; \\
48^{\circ} 53^{\prime} \mathrm{N} \& 165^{\circ} 07^{\prime} \mathrm{E}(100) ; \\
47^{\circ} 12^{\prime} \mathrm{N} \& 165^{\circ} 29^{\prime} \mathrm{E}(100)\end{array}$ \\
\hline \multicolumn{3}{|l|}{ Lamniformes } \\
\hline $\begin{array}{l}\text { Lamna ditropis Hubbs et } \\
\text { Follett, } 1947 / 131.0 \mathrm{~cm}, 24.56 \\
\mathrm{~kg}\end{array}$ & 1 & $42^{\circ} 35^{\prime} \mathrm{N} \& 148^{\circ} 50^{\prime} \mathrm{E}(1)$ \\
\hline \multicolumn{3}{|l|}{ Carcharhiniformes } \\
\hline $\begin{array}{l}\text { Prionace glauca (Linnaeus, } \\
1758) / 140.0 \mathrm{~cm}, 42 \mathrm{~kg}\end{array}$ & 1 & $41^{\circ} 29^{\prime} \mathrm{N} \& 147^{\circ} 16^{\prime} \mathrm{E}(1)$ \\
\hline
\end{tabular}

net bag. Locations of the trawls are given in Table 1.

All specimens were examined macroscopically for the presence of ectoparasites immediately after capture and then dissected and studied for endoparasitic helminths and other metazoan parasites using a standard method of parasitological examination (Bykhovskaya-Pavlovskaya, 1985). All sharks caught alive were released after measuring. Only two specimens were hauled, already dead, and subsequently included in the parasitological survey. Prefixation treatment of trematodes and cestodes included cleaning and straightening for better identification. Acanthocephala were transferred to fresh water until the proboscis everted prior to fixation. All parasites except nematodes were preserved in $70 \%$ and $96 \%$ ethanol. Nematodes were preserved in $4 \%$ formaldehyde, and three days later, transferred to $70 \%$ ethanol for longterm storage. Subsequently, temporary glycerol preparations of acanthocephalans and nematodes were made. Digenea were stained with acetic carmine, dehydrated, contrasted (cleared) with dimethyl phthalate, and finally mounted in Canada balsam. Cestoda were hydrated, stained with Harris's hematoxylin, differentiated in tap water, de-stained in ethanol, dehydrated, cleaned in methyl salicylate, and finally mounted in Canada balsam (Jensen et al., 2011).
In consideration of how to present our findings, we faced difficulties due to the fact that specimens of some species were caught with a significant difference in time and location. However, taking into account that almost all captures occurred within one month and the fact that in the open ocean environment, millions of square kilometres can be regarded as homogeneous from a biological point of view, in Table 2 we have provided the infection indices all together (all specimens of one species were pooled together to count parasitological indices), calculated in accordance with Bush et al. (1999). However, in Table 1, we have provided a list of the examined species and the exact catch locations, with the number of specimens caught.

\section{Results}

Helminths were found in all studied fish species except slender barracudina, Lestidiops ringens (Jordan, Gilbert, 1880), and scaly paperbone, Scopelosaurus harryi (Mead, 1953). Most species were infected by the larvae of $P$. speciosum and Anisakis sp. juveniles (Tables 2-8). Unlike all other bony fishes studied by us, M. atlantica and long snouted lancetfish, Alepisaurus ferox Lowe, 1833, had in their intestines both undeveloped (normal) plerocercoids $P$. speciosum, up to $3 \mathrm{~cm}$ long, and large plero- 
Table 2. Infection of Pacific salmon. Таблица 2. Паразиты тихоокеанских лососей.

\begin{tabular}{|c|c|c|c|c|c|}
\hline \multicolumn{2}{|c|}{ Parasite } & $\begin{array}{l}\text { Dev. } \\
\text { stage }\end{array}$ & $\begin{array}{c}\text { Prevalence } \\
(\%)\end{array}$ & $\begin{array}{l}\text { Intensity } \\
\text { (mean) }\end{array}$ & $\begin{array}{c}\text { Site of } \\
\text { infection }\end{array}$ \\
\hline \multicolumn{6}{|c|}{ Oncorhynchus gorbuscha $(\mathrm{n}=15)$} \\
\hline Cestoda & $\begin{array}{l}\text { Pelichnibothrium } \\
\text { speciosum }\end{array}$ & larva & 100.00 & $1-36(10.07)$ & guts \\
\hline Copepoda & $\begin{array}{c}\text { Lepeophtheirus } \\
\text { salmonis salmonis }\end{array}$ & ad. & 26.67 & $1-5(2.25)$ & $\begin{array}{c}\text { body } \\
\text { surface }\end{array}$ \\
\hline \multirow{3}{*}{ Chromadorea } & Anisakis sp. & juv. III & 33.33 & $1-13(4.40)$ & guts \\
\hline & $\begin{array}{l}\text { Hysterothylacium } \\
\text { sp. }\end{array}$ & juv. III & 6.66 & $1(1.00)$ & guts \\
\hline & Ascarophis sp. & subad. & 6.66 & $1(1.00)$ & guts \\
\hline $\begin{array}{l}\text { Palaeacantho- } \\
\text { cephala }\end{array}$ & $\begin{array}{c}\text { Echinorhynchus } \\
\text { salmonis }\end{array}$ & ad. & 6.66 & $1(1.00)$ & intestine \\
\hline \multicolumn{6}{|c|}{ Oncorhynchus keta $(\mathrm{n}=15)$} \\
\hline Copepoda & $\begin{array}{c}\text { Lepeophtheirus } \\
\text { salmonis salmonis }\end{array}$ & ad. & 20.00 & $1-3(2.00)$ & $\begin{array}{l}\text { body } \\
\text { surface }\end{array}$ \\
\hline \multirow{3}{*}{ Cestoda } & $\begin{array}{l}\text { Eubothrium } \\
\text { salvelini }\end{array}$ & ad. & 26.67 & $1(1.00)$ & guts \\
\hline & $\begin{array}{l}\text { Pelichnibothrium } \\
\text { speciosum }\end{array}$ & larva & 100.00 & $4-56(26.47)$ & guts \\
\hline & $\begin{array}{c}\text { Nybelinia } \\
\text { surmenicola }\end{array}$ & larva & 6.67 & $2(2.00)$ & $\begin{array}{c}\text { stomach } \\
\text { wall }\end{array}$ \\
\hline Chromadorea & Anisakis sp. & juv. III & 20.00 & $2-10(5.67)$ & guts \\
\hline \multicolumn{6}{|c|}{ Oncorhynchus kisutch $(\mathrm{n}=3)$} \\
\hline Cestoda & $\begin{array}{c}\text { Pelichnibothrium } \\
\text { speciosum }\end{array}$ & ad. & 100.00 & $4-56(26.47)$ & guts \\
\hline
\end{tabular}

cercoids, up to $15 \mathrm{~cm}$ long with undeveloped sexual complexes (Fig. 1).

\section{Discussion}

As can be seen in Tables 2-8, species richness of macroparasites in pelagic fish in the north-western Pacific is quite poor, but studied specimens are highly infected. Well-known generalists in the area (Volkov et al., 1999), anisakid and raphidascaridid juveniles, plerocercoids of $N$. surmenicola, and E. gadi made up $13 \%$ of the total number of parasites found in bony fishes and $22.2 \%$ of all species. Another common tapeworm, $P$. speciosum, could be labelled as a generalist because plerocercoids of this species made up $64 \%$ of the total number of parasites and were found infecting 16 out of 23 examined teleost fish. Klimpel et al. (2006) studied the infection of bony fishes and revealed that in the Greenland Sea and in the Irminger Sea, the diversity of parasites in pelagic fish species was poor, while the parasite load in demersal fishes was heavy. Species diversity of marine fish parasites depends on the specific feeding behaviour of the hosts, the availability of intermediate and final hosts, depth distribution and host migration. Similarities in diet and habitat of potential host species often result in a similar or even identical parasite fauna (Dogiel, 
Table 3. Infection of Perciformes fish examined. Таблица 3. Зараженность представителей отряда Perciformes.

\begin{tabular}{|c|c|c|c|c|c|}
\hline \multicolumn{2}{|c|}{ Parasite } & $\begin{array}{l}\text { Dev. } \\
\text { stage }\end{array}$ & $\begin{array}{c}\text { Prevalence } \\
(\%)\end{array}$ & $\begin{array}{c}\text { Intensity } \\
\text { (mean) }\end{array}$ & $\begin{array}{c}\text { Site of } \\
\text { infection }\end{array}$ \\
\hline \multicolumn{6}{|c|}{ Hyperoglyphe japonica $(\mathrm{n}=2)$} \\
\hline Cestoda & $\begin{array}{l}\text { Glossobothrium } \\
\text { nipponicum }\end{array}$ & adult & 100.00 & $10.07(1-36)$ & intestine \\
\hline \multicolumn{6}{|c|}{ Brama japonica $(\mathrm{n}=13)$} \\
\hline \multirow{2}{*}{ Cestoda } & $\begin{array}{c}\text { Nybelinia } \\
\text { surmenicola }\end{array}$ & larva & 7.69 & $1(1.00)$ & $\begin{array}{c}\text { stomach } \\
\text { wall }\end{array}$ \\
\hline & $\begin{array}{l}\text { Pelichnibothrium } \\
\text { speciosum }\end{array}$ & larva & 69.32 & $3-14(6.67)$ & intestine \\
\hline $\begin{array}{c}\text { Chroma- } \\
\text { dorea }\end{array}$ & $\begin{array}{l}\text { Hysterothylacium } \\
\text { sp. }\end{array}$ & juv. III & 7.69 & $1(1.00)$ & guts \\
\hline \multicolumn{6}{|c|}{ Icichthys lockingtoni $(\mathrm{n}=2)$} \\
\hline \multirow{2}{*}{ Trematoda } & $\begin{array}{l}\text { Licithophyllum } \\
\text { botryoporon }\end{array}$ & ad. & 50.00 & $1(1.00)$ & guts \\
\hline & $\begin{array}{c}\text { Paraccacladium cf. } \\
\text { jamiesoni }\end{array}$ & ad. & 50.00 & $1(1.00)$ & guts \\
\hline Cestoda & $\begin{array}{l}\text { Pelichnibothrium } \\
\text { speciosum }\end{array}$ & larva & 50.00 & $1(1.00)$ & guts \\
\hline \multicolumn{6}{|c|}{ Zaprora silenus $(\mathrm{n}=6)$} \\
\hline Trematoda & $\begin{array}{l}\text { Prodistomum } \\
\text { alaskense }\end{array}$ & ad. & 66.67 & $5-94(42.50)$ & intestine \\
\hline Cestoda & $\begin{array}{l}\text { Pelichnibothrium } \\
\text { speciosum }\end{array}$ & larva & 66.67 & $2-9(5.50)$ & intestine \\
\hline $\begin{array}{l}\text { Chroma- } \\
\text { dorea }\end{array}$ & $\begin{array}{l}\text { Hysterothylacium } \\
\text { sp. }\end{array}$ & juv. III & 16.67 & $1(1.00)$ & guts \\
\hline
\end{tabular}

1962). In contrast, hosts with different food preferences may have large differences in their parasite fauna (Klimpel et al., 2006). Within the north-western Pacific, a combination of different factors, especially the availability of intermediate hosts and the habitat preferences of the fish, can be seen as the main reason for the observed infection.

All studied species of bony fish could be divided into two unequal groups: eurybathic species that perform significant vertical migrations (lumpfish Aptocyclus ventricosus, prowfish Zaprora silenus, northern smoothtongue Leuroglossus schmidti, and medusafish Icichthys lockingtoni) and epipelagic anadromous and non-anadromous species that inhabit cold and moderate waters of the north-western Pacific Ocean (all other species). Infection of eurybathic species differs from that of other fish due to the presence of trematodes.

Our data on the infection of the poorly studied lumpfish, Aptocyclus ventricosus, is similar to the data of Machida (1985) who performed a targeted study on this species off northern Japan. However, our specimens were collected to the north closer to the Bering Sea, so in our study infection by digenean species is presented by more polar species, like Prodistomum alaskense (Ward et Fillingham, 1934). Prosorhynchus mizellei Kruse, 1977 was described from $A$. ventricosus caught in the Bering Sea (Kruse, 1977), but that seems to be the only record of $P$. 
Table 4. Infection of Aulopiformes. Таблица 4. Зараженность представителей отряда Aulopiformes.

\begin{tabular}{|c|c|c|c|c|c|}
\hline \multicolumn{2}{|c|}{ Parasite } & $\begin{array}{l}\text { Dev. } \\
\text { stage }\end{array}$ & $\begin{array}{c}\text { Prevalence } \\
\text { (\%) }\end{array}$ & $\begin{array}{c}\text { Intensity } \\
\text { (mean) }\end{array}$ & $\begin{array}{c}\text { Site of } \\
\text { infection }\end{array}$ \\
\hline \multicolumn{6}{|c|}{ Alepisaurus ferox $(\mathrm{n}=4)$} \\
\hline \multirow{2}{*}{ Cestoda } & $\begin{array}{c}\text { Pelichnibothrium } \\
\text { speciosum }\end{array}$ & larva & 100.00 & $\begin{array}{c}60-211 \\
(122.75)\end{array}$ & guts \\
\hline & $\begin{array}{c}\text { Nybelinia } \\
\text { surmenicola }\end{array}$ & larva & 50.00 & $\begin{array}{c}16-28 \\
(22.00) \\
\end{array}$ & $\begin{array}{c}\text { stomach } \\
\text { wall }\end{array}$ \\
\hline \multirow{2}{*}{ Chromadorea } & Anisakis sp. & $\begin{array}{l}\text { juv. } \\
\text { III }\end{array}$ & 50.00 & $4-7(5.50)$ & guts \\
\hline & $\begin{array}{l}\text { Hysterothylacium } \\
\text { sp. }\end{array}$ & $\begin{array}{l}\text { juv. } \\
\text { III }\end{array}$ & 25.00 & $2(2.00)$ & guts \\
\hline \multicolumn{6}{|c|}{ Anotopterus nikparini $(\mathrm{n}=9)$} \\
\hline Cestoda & $\begin{array}{c}\text { Pelichnibothrium } \\
\text { speciosum }\end{array}$ & larva & 11.00 & $4(4.00)$ & guts \\
\hline \multicolumn{6}{|c|}{ Magnisudis atlantica $(\mathrm{n}=1)$} \\
\hline Cestoda & $\begin{array}{c}\text { Pelichnibothrium } \\
\text { speciosum }\end{array}$ & larva & in 1 of 1 & $26(26.00)$ & guts \\
\hline
\end{tabular}

Table 5. Infection of Myctophiformes. Таблица 5. Зараженность представителей отряда Myctophiformes.

\begin{tabular}{|c|c|c|c|c|c|}
\hline \multicolumn{2}{|c|}{ Parasite } & $\begin{array}{l}\text { Dev. } \\
\text { stage }\end{array}$ & $\begin{array}{c}\text { Prevalence } \\
(\%)\end{array}$ & $\begin{array}{l}\text { Intensity } \\
\text { (mean) }\end{array}$ & $\begin{array}{c}\text { Site of } \\
\text { infection }\end{array}$ \\
\hline \multicolumn{6}{|c|}{ Diaphus theta $(\mathrm{n}=25)$} \\
\hline Cestoda & $\begin{array}{l}\text { Hysterothylacium } \\
\text { sp. }\end{array}$ & larva & 4.00 & $1(1.00)$ & guts \\
\hline Chromadorea & Anisakis sp. & juv. III & 4.00 & $1(1.00)$ & guts \\
\hline \multicolumn{6}{|c|}{ Notoscopelus japonicus $(\mathrm{n}=34)$} \\
\hline \multirow{2}{*}{ Chromadorea } & Anisakis sp. & larva & 14.71 & $1-2(1.20)$ & guts \\
\hline & Ascarophis sp. & subad. & 2.94 & $1(1.00)$ & guts \\
\hline \multicolumn{6}{|c|}{ Stenobrachius leucopsarus $(\mathrm{n}=14)$} \\
\hline Chromadorea & Anisakis sp. & juv. III & 14.29 & $1(1.00)$ & guts \\
\hline \multicolumn{6}{|c|}{ Tarletonbeania crenularis $(\mathrm{n}=1)$} \\
\hline Cestoda & $\begin{array}{l}\text { Pelichnibothrium } \\
\text { speciosum }\end{array}$ & larva & in 1 of 1 & $1(1.00)$ & guts \\
\hline
\end{tabular}

mizellei. Prowfish Z. silenus has never been studied for parasitic infection, so all three common species are recorded for the first time. Northern smoothtongue $L$. schmidti has been studied, but quite fragmentarily. It was recorded as a host of Anisakis sp. (Solovyova, 1999), Steringophorus congeri Shen, 1987, and Aponurus argentini Polyanski, 1952 (see 
Table 6. Infection of Scorpaeniformes, Scombriformes, and Beloniformes. Таблица 6. Зараженность представителей отрядов Scorpaeniformes, Scombriformes и Beloniformes.

\begin{tabular}{|c|c|c|c|c|c|}
\hline \multicolumn{2}{|c|}{ Parasite } & $\begin{array}{l}\text { Dev. } \\
\text { stage }\end{array}$ & $\begin{array}{c}\text { Preva- } \\
\text { lence }(\%)\end{array}$ & $\begin{array}{c}\text { Intensity } \\
\text { (mean) }\end{array}$ & $\begin{array}{c}\text { Site of } \\
\text { infection }\end{array}$ \\
\hline \multicolumn{2}{|c|}{1} & 2 & 3 & 4 & 5 \\
\hline \multicolumn{6}{|c|}{ Scomber japonicus $(\mathrm{n}=25)$} \\
\hline \multirow{2}{*}{ Chromadorea } & Anisakis sp. & juv. III & 64.00 & $1-21(7.25)$ & guts \\
\hline & $\begin{array}{l}\text { Hysterothylacium } \\
\text { sp. }\end{array}$ & juv. III & 20.00 & $1-35(8.20)$ & guts \\
\hline \multirow{3}{*}{ Trematoda } & $\begin{array}{c}\text { Prodistomum } \\
\text { orientale }\end{array}$ & ad. & 12.00 & $5-67(32.00)$ & intestine \\
\hline & Opechona olssoni & ad. & 4.00 & $1(1.00)$ & intestine \\
\hline & $\begin{array}{l}\text { Lecithocladium } \\
\text { excisum }\end{array}$ & ad. & 44.00 & $1-5(1.82)$ & intestine \\
\hline \multirow{3}{*}{$\begin{array}{l}\text { Palaeacantho- } \\
\text { cephala }\end{array}$} & $\begin{array}{c}\text { Rhadinorhynchus } \\
\text { trachuri }\end{array}$ & ad. & 12.00 & $1(1.00)$ & intestine \\
\hline & Rh. cololabis & ad. & 32.00 & $1-2(1.14)$ & intestine \\
\hline & Rh. selkirki & ad. & 4.00 & $1(1.00)$ & intestine \\
\hline Cestoda & $\begin{array}{c}\text { Pelichnibothrium } \\
\text { speciosum }\end{array}$ & larva & 16.00 & $1-2(1.50)$ & guts \\
\hline \multicolumn{6}{|c|}{ Aptocyclus ventricosus $(n=15)$} \\
\hline \multirow{2}{*}{ Trematoda } & $\begin{array}{c}\text { Prodistomum } \\
\text { alaskense }\end{array}$ & ad. & 26.67 & $3-11(6.00)$ & intestine \\
\hline & $\begin{array}{l}\text { Paraccacladium } \\
\text { cf. jamiesoni }\end{array}$ & ad. & 73.33 & $1-15(5.54)$ & intestine \\
\hline \multirow{3}{*}{ Chromadorea } & Ascarophis sp. & subad. & 26.67 & $1-2(1.50)$ & guts \\
\hline & Anisakis sp. & juv. III & 6.67 & $1(1.00)$ & guts \\
\hline & $\begin{array}{l}\text { Hysterothylacium } \\
\text { sp. }\end{array}$ & juv. III & 6.67 & $1(1.00)$ & guts \\
\hline Acanth. & $\begin{array}{l}\text { Echinorhynchus } \\
\text { gadi }\end{array}$ & ad. & 6.67 & $1(1.00)$ & intestine \\
\hline \multirow{2}{*}{ Cestoda } & $\begin{array}{c}\text { Nybelinia } \\
\text { surmenicola }\end{array}$ & larva & 6.67 & $1(1.00)$ & $\begin{array}{c}\text { stomach } \\
\text { wall }\end{array}$ \\
\hline & $\begin{array}{c}\text { Pelichnibothrium } \\
\text { speciosum }\end{array}$ & larva & 6.67 & $1(1.00)$ & guts \\
\hline \multicolumn{6}{|c|}{ Cololabis saira $(\mathrm{n}=52)$} \\
\hline Chromadorea & Anisakis sp. & juv. III & 7.69 & $1-7(2.50)$ & guts \\
\hline Copepoda & Penella sp. & ad. & 23.08 & $1-4(1.75)$ & $\begin{array}{c}\text { body } \\
\text { surface, } \\
\text { musculature }\end{array}$ \\
\hline
\end{tabular}


Table 6 (continued). Таблица 6 (продолжение).

\begin{tabular}{|c|c|c|c|c|c|}
\hline & $\mathbf{1}$ & $\mathbf{2}$ & $\mathbf{3}$ & $\mathbf{4}$ & $\mathbf{5}$ \\
\hline \multirow{2}{*}{$\begin{array}{c}\text { Palaeacantho- } \\
\text { cephala }\end{array}$} & $\begin{array}{c}\text { Rhadinorhynchus } \\
\text { cololabis }\end{array}$ & ad. & 98.08 & $1-8(3.07)$ & intestine \\
\cline { 2 - 6 } & Rh. selkirki & ad. & 3.85 & $1(1.00)$ & intestine \\
\cline { 2 - 6 } & Rh. trachuri & ad. & 5.77 & $1(1.00)$ & intestine \\
\hline Cestoda & $\begin{array}{c}\text { Pelichnibothrium } \\
\text { speciosum }\end{array}$ & larva & 26.92 & $1-3(1.71)$ & guts \\
\hline
\end{tabular}

Table 7. Infection of other species of bony fishes. Таблица 7. Зараженность представителей других отрядов костистых рыб.

\begin{tabular}{|c|c|c|c|c|c|}
\hline \multicolumn{2}{|c|}{ Parasite } & $\begin{array}{l}\text { Dev. } \\
\text { stage }\end{array}$ & $\begin{array}{c}\text { Prevalence } \\
(\%)\end{array}$ & $\begin{array}{c}\text { Intensity } \\
\text { (mean) }\end{array}$ & $\begin{array}{c}\text { Site of } \\
\text { infection }\end{array}$ \\
\hline \multicolumn{6}{|c|}{ Sardinops sagax melanostictus $(\mathrm{n}=10)$} \\
\hline Chromadorea & Anisakis sp. & $\begin{array}{l}\text { juv. } \\
\text { III }\end{array}$ & 10.00 & $1(1.00)$ & guts \\
\hline \multicolumn{6}{|c|}{ Leuroglossus schmidti $(\mathrm{n}=16)$} \\
\hline Cestoda & $\begin{array}{l}\text { Pelichnibothrium } \\
\text { speciosum }\end{array}$ & larva & 6.25 & $1(1.00)$ & guts \\
\hline \multirow{2}{*}{ Trematoda } & $\begin{array}{l}\text { Licithophyllum } \\
\text { botryoporon }\end{array}$ & ad. & 6.25 & $1(1.00)$ & intestine \\
\hline & $\begin{array}{c}\text { Paraccecladium } \\
\text { jamiesoni }\end{array}$ & $\mathrm{ad}$, & 12.50 & $1-2(1.50)$ & intestine \\
\hline \multicolumn{6}{|c|}{ Desmodema lorum $(\mathrm{n}=1)$} \\
\hline \multirow{2}{*}{ Chromadorea } & $\begin{array}{l}\text { Hysterothylacium } \\
\text { sp. }\end{array}$ & $\begin{array}{l}\text { juv. } \\
\text { III }\end{array}$ & in 1 of 1 & $1(1.00)$ & guts \\
\hline & Anisakis sp. & $\begin{array}{l}\text { juv. } \\
\text { III }\end{array}$ & in 1 of 1 & $6(6.00)$ & guts \\
\hline Cestoda & $\begin{array}{c}\text { Pelichnibothrium } \\
\text { speciosum }\end{array}$ & larva & in 1 of 1 & $10(10.00)$ & guts \\
\hline \multicolumn{6}{|c|}{ Gasterosteus aculeatus $(\mathrm{n}=214)$} \\
\hline Chromadorea & Anisakis sp. & $\begin{array}{c}\text { juv. } \\
\text { III }\end{array}$ & 2.34 & $1-2(1.40)$ & guts \\
\hline \multirow{2}{*}{ Cestoda } & $\begin{array}{c}\text { Pelichnibothrium } \\
\text { speciosum }\end{array}$ & larva & 7.01 & $1(1.00)$ & guts \\
\hline & $\begin{array}{c}\text { Bothriocephalus } \\
\text { scorpii }\end{array}$ & larva & 7.01 & $1-3(1.25)$ & guts \\
\hline Trematoda & $\begin{array}{c}\text { Bunodera } \\
\text { mediovitellata }\end{array}$ & $\mathrm{ad}$. & 12.62 & $1-3(1.13)$ & intestine \\
\hline
\end{tabular}

Kuramochi, 2009). All three species mentioned in Table 7 are noted for L. schmidti for the first time. The same is true for the three parasitic species that we found in medusafish I. lockingtoni, which seems to have never been checked for infection before. 
Table 8. Infection of cartilaginous fish. Таблица 8. Зараженность хрящевых рыб.

\begin{tabular}{|c|c|c|c|c|c|}
\hline \multicolumn{2}{|c|}{ Parasite } & $\begin{array}{l}\text { Dev. } \\
\text { stage }\end{array}$ & $\begin{array}{c}\text { Prevalence } \\
(\%)\end{array}$ & $\begin{array}{c}\text { Intensity } \\
\text { (mean) }\end{array}$ & $\begin{array}{c}\text { Site of } \\
\text { infection }\end{array}$ \\
\hline \multicolumn{6}{|c|}{ Lamna ditrops $(\mathrm{n}=1)$} \\
\hline Chromadorea & Anisakis sp. & juv. III & in 1 of 1 & $7(7.00)$ & stomach \\
\hline Trematoda & $\begin{array}{c}\text { Lecithocladium } \\
\text { excisum }\end{array}$ & ad. & in 1 of 1 & $1(1.00)$ & stomach \\
\hline Cestoda & $\begin{array}{c}\text { Nybelinia } \\
\text { surmenicola }\end{array}$ & ad. & in 1 of 1 & $27(27.00)$ & $\begin{array}{l}\text { spiral } \\
\text { valve }\end{array}$ \\
\hline Cestoda & $\begin{array}{c}\text { Dinobothrium } \\
\text { sp. }\end{array}$ & ad. & in 1 of 1 & $2(2.00)$ & $\begin{array}{l}\text { spiral } \\
\text { valve }\end{array}$ \\
\hline \multicolumn{6}{|c|}{ Prionace glauca $(\mathrm{n}=1)$} \\
\hline Chromadorea & Anisakis sp. & juv. III & in 1 of 1 & $6(6.00)$ & stomach \\
\hline \multirow{3}{*}{ Cestoda } & $\begin{array}{c}\text { Platybothrium } \\
\text { auriculatum }\end{array}$ & ad. & in 1 of 1 & $\begin{array}{c}323 \\
(323.00) \\
\end{array}$ & $\begin{array}{l}\text { spiral } \\
\text { valve }\end{array}$ \\
\hline & $\begin{array}{c}\text { Anthobothrium } \\
\text { caseyi }\end{array}$ & ad. & in 1 of 1 & $\begin{array}{c}120 \\
(120.00)\end{array}$ & $\begin{array}{l}\text { spiral } \\
\text { valve }\end{array}$ \\
\hline & $\begin{array}{l}\text { Scyphophylli- } \\
\text { dium exiguum }\end{array}$ & ad. & in 1 of 1 & $\begin{array}{c}155 \\
(155.00) \\
\end{array}$ & $\begin{array}{l}\text { spiral } \\
\text { valve }\end{array}$ \\
\hline
\end{tabular}

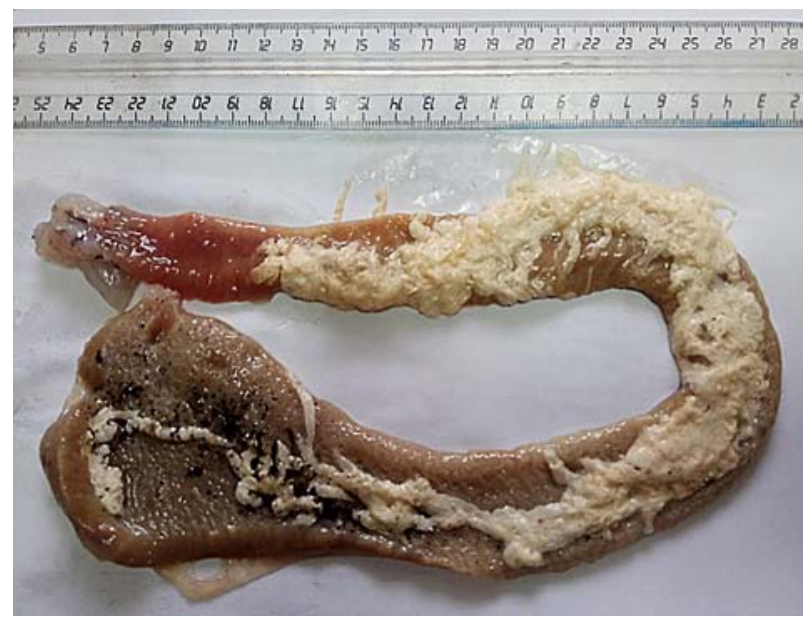

Fig. 1. Dissected intestine of Alepisaurus ferox, infected by plerocercoids of Pelichnibothrium speciosum. Рис. 1. Вскрытый кишечник Alepisaurus ferox, зараженного плероцеркоидами Pelichnibothrium speciosum.

The infection of Pacific salmon is a wellstudied issue. Many publications dedicated to the parasites of salmon at different developing stages are available (e.g. Konovalov, 1971; Karmanova, 1998; Vyalova, 2002; Pospehov et al., 2014). Pelichnibothrium speciosum (Monticelli, 1889) was the most abundant species in our samples. For the first time, phyllobothriidean plerocercoids were found in Pacific salmon by F. Zschokke and described as Pelichniboth- 
rium caudatum Zschokke in Zschokke and Heitz, 1914 (see Zschokke \& Heitz, 1914). Zmeev (1936) moved this species to the genus Phyllobothrium Van Beneden, 1850. Dubinina (1971) considered $P$. caudatum as conspecific with $P$. speciosum. However, Szholz et al. (1998) disagreed with this, based on the presence in the zone of proglottis differentiation situated far posterior to the scolex of $P$. speciosum sensu Dubinina, 1971. Most authors indicate the common plerocercoids from Pacific salmon as $P$. speciosum (Vyalova, 2002; Karmanova, 1998; Pospehov et al., 2014). The type host of $P$. speciosum is $A$. ferox (see Monticelli, 1889). In our materials collected from nine examined specimens of $A$. ferox, we found both small plerocercoids $(2-3 \mathrm{~cm}$, matching the description of $P$. caudatum) and large (up to $15 \mathrm{~cm}$ ), as described by Szholtz et al. (1998). Since we could observe gradual change in the scolex morphology and were convinced that the large plerocercoids processed are conspecific with the small ones we found in 16 out of 23 examined teleost species, including $A$. ferox, all of them in Tables $2-8$ are indicated as $P$. speciosum.

In the works of Mamaev et al. (1959) and Margolis (1963) were records of Pacific salmon species in the open ocean being heavily infected by marine digenean species (Brachyphallus crenatus (Rudolphi, 1802) and others), but in our research, we found none of them. It can be assumed that this is a consequence of the wavelike change in the number of both parasites and their hosts. In 1958, when Mamaev and his colleagues collected their samples, only 39 tons of pink salmon were caught in the Russian Far East, while in 2018, it was the historical maximum of the pink salmon fishing season, and more than 511 tons were caught (Gordeev et al., 2018). Undoubtedly, the 13-fold difference in the number of hosts that did not form shoals during the feeding migration could affect the dynamics of parasitic cycles.

Pacific barrelfish, Hyperoglyphe japonica (Döderlein, 1884), was heavily infected solely by a cestode Glossobothrium nipponicum Yamaguti, 1952 (Table 3). Taking into account that this fish feeds on Japanese anchovy, Engraulis japonicas Temminck et Schlegel, 1846, euphasiids, hyperiids, and squid, as do many other teleost fish (Chuchukalo, 2006), host specificity of this tapeworm seems to be limited to a small number of fish hosts, including Seriolella brama (Günther, 1860) (see Gulyaev, Korotaeva, 1989) and Schedophilus velaini (Sauvage, 1879) (see Brabec et al., 2015). Another representative of the order Perciformes - Pacific pomfret, Brama japonica Hilgendorf, 1878, is well studied in terms of parasitic infection by: copepods (McDonald, Margolis, 1995), digeneans (Love, Moser, 1983; Moles, 2007), cestodes (Iannacone, Alvariño, 2013), and acanthocephalans (Didenko, Shevshenko, 1999) throughout its wide habitat range. However, $P$. speciosum and Hysterothylacium sp. are recorded as infecting for the first time.

Two species of Aulopiformes - North Pacific daggertooth, Anotopterus nikparini Kukuev, 1998, and A. ferox - are similar both in appearance and in the structure of the jaw apparatus. However, A. nikparini bites pieces of flesh from its victims, while $A$. ferox swallows its prey whole. This dissimilarity in the feeding method is reflected in the dramatic difference in the helminth infection: only one out of the nine examined specimens of $A$. nikparini was infected by four plerocercoids of $P$. speciosum, while A. ferox was heavily infected by generalist nematodes and especially cestodes (Table 4, Fig. 1). The reason why $P$. speciosum develops into a large plerocercoid only in A. ferox and M. atlantica among fish species remains unclear. $M$. atlantica was studied for infection for the first time.

Mictophids California headlightfish, Diaphus theta Eigenmann et Eigenmann, 1890, Japanese lanternfish Notoscopelus japonicus (Tanaka, 1908), northern lampfish, Stenobrachius leucopsarus (Eigenmann et Eigenmann, 1890), and blue lanternfish, Tarletonbeania crenularis (Jordan et Gilbert, 1880), showed weak involvement in the parasitic life cycles. Only 10 out of 74 fishes were infected by generalists - Anisakis sp., Ascarophis sp., and by P. speciosum (Table 5). 
Chub mackerel, Scomber japonicus Houttuyn, 1782, is well studied for parasitic infection in the north-western Pacific Ocean (Ishihara, 1968; Kovalenko, 1986) and the biological diversity of its infection is the greatest among epipelagic species covered in this research, due to its significant migrations from the areas of spawning (Japanese archipelago) to the feeding area in the more productive north areas. All parasitic species found by us were previously recorded repeatedly in chub mackerel (Volkov et al., 1999).

Infection of the Pacific saury, Cololabis saira (Brevoort, 1856), is well studied in the area under consideration (Kurochkin etal., 1987; Gordeev et al., 2017; Suyama et al., 2019). Our findings are broadly consistent with the available information on infection of C. saira, in the northern part of the Pacific Ocean (Table 6). Total infection by acanthocephalans of the genus Rhadinorhynchus Lühe, 1911 was characterised by higher prevalence of Rhadinorhynchus cololabis Laurs et McCauley, 1964, while in more southerly waters, Rhadinorhynchus trachuri Harada, 1935 prevailed in the Pacific saury's intestine.

Japanese pilchard, Sardinops sagax melanosticta (Temminck et Schlegel, 1846), and whiptail ribbonfish, Desmodema lorum Rosenblatt et Butler, 1977, were poorly observed in this work. However, in the latter fish species, we have found Hysterothylacium sp., Anisakis sp., and $P$. speciosum (Table 7). This is the first study of whiptail ribbonfish infection.

Threespine stickleback, G. aculeatus, an anadromous species in the Pacific Ocean, performs significant migrations from its spawning grounds to the open waters. Our specimens were caught at a distance of 500-600 km from the shore and were infected only by Anisakis sp., $P$. speciosum, Bothriocephalus scorpii (Müller, 1776) and Bunodera mediovitellata Zymbaluk et Roitman, 1966. Among the listed parasites, only $B$. mediovitellata remained in the examined sticklebacks from the fresh waters since its first intermediate hosts are insects (Trichoptera) (Caira, 1981). Our study showed that G. aculeatus's infection significantly differs from all oth- er studied species of bony fish. The stickleback feeds in the sea; however, the parasitological examination of more than 200 specimens revealed the prevalence of coastal (B. scorpii) and freshwater (B. mediovitellata) parasites. This is the first data on G. aculeatus infection so far in the open ocean.

The salmon shark, Lamna ditropis Hubbs et Follett, 1947, was studied in a single specimen and comparatively little infection was found in the form of the adult stages of $N$. surmenicola and Dinobothrium sp. (Table 8). Anisakis sp. and Lecithocladium excisum (Rudolphi, 1819) were found in the stomach. Lamna ditrops is a paratenic host of Anisakis sp., while for $L$. excisum it is a postcyclic host. Both cestodes were previously recorded in the salmon shark by Palm (2004). Two specimens of Dinobothrium found by us do not fit the morphological description of any of the six known species and probably belong to a new species. The blue shark, Prionace glauca (Linnaeus, 1758), was studied in a single specimen too and was not much older than the salmon shark specimen, but was much more heavily infected. All three species of cestodes recorded from the spiral valve were already recorded in P. glauca (MerloSerna, Garcia-Prieto, 2016; Alves et al., 2017).

Both species of sharks studied feed on fish. However, the blue sharks mostly feed on Leuroglossus schmidti, Cololabis saira, and myctophids with sea elephants, planktonic crustaceans, thaliaceans, chum salmon, and coho salmon being found occasionally. In the feeding of the salmon shark, pink salmon, and other salmon, Engraulis japonicus, Sardinops sagax melanostictus, Clupea pallasii, and squid prevail (Chuchukalo, 2006). Despite their generally different feeding habits, both species of shark share the same feeding base, so in our opinion, the clear distinction between infections by cestodes arose due to the significant difference in the morphology of the spiral valve (Leigh et al., 2019). The blue shark is a representative of the order Carcharhiniformes and has a cylindrical spiral in the valve, while the salmon shark belongs to the order Lamniformes and has a vertical spiral in the valve. 


\section{Acknowledgements}

The authors with to thank the crew of the RV Professor Kaganosky and all members of the ichthyologic team - Drs. Valery Shevlyakov and Starovoytov Alexander, and also Pavel Milovankin, Elena Degtyareva, Sergey Ponomarev (Pacific Branch of the Russian Federal Research Institute of Fisheries and Oceanography, Vladivostok) for the help with sampling, Dr. Tatyana Polyakova (A.O. Kovalevsky Institute of Biology of the Southern Seas of RAS) for the help with cestodes identification, and Dr. Alexey Orlov (Russian Federal Research Institute of Fisheries and Oceanography, Moscow) for essential advices.

\section{Funding}

The work was supported by the Russian Science Foundation, grant No.17-74-10203.

\section{Compliance with ethical standards}

Conflict of Interest: The authors declare that they have no conflict of interest.

Ethical approval: This article does not contain any studies with animals performed by any of the authors.

Sampling and field studies: All necessary permits for sampling and observational field studies have been obtained by the authors from the competent authorities and are mentioned in the acknowledgements, if applicable.

\section{References}

Alves P.V., de Chambrier A., Scholz T. Luque J.L. 2017. Annotated checklist of fish cestodes from South America // Zookeys. Vol.650. P.1-205. doi: 10.3897/zookeys.650.10982

Brabec J., Waeschenbach A., Scholz T., Littlewood D.T.J., Kuchta R. 2015. Molecular phylogeny of the Bothriocephalidea (Cestoda): molecular data challenge morphological classification // International Journal for Parasitology. Vol.45. No.12.P.761-771. doi: 10.1016/ j.ijpara.2015.05.006

Rohde K. (ed.) 2005. Marine parasitology. Collingwood. CSIRO Publishing (Commonwealth Scientific and Industrial Research Organization). 565 p.

Bush A.O., Lafferty K.D., Lotz J.M., Shostak A.W. 1997. Parasitology meets ecology on its own terms: Margolis et al. revisited // The Journal of Parasitology. Vol.83. No.3. P.575-583. doi: 10.2307/3284227

Bykhovskaya-Pavlovskaya I.E. 1985. [Parasites of fishes. The Manual.]. Leningrad. Nauka. 124 p. [In Russian]
Caira J.N. 1981. Parasitism of Trichoptera by Bunodera mediovitellata (Digenea: Allocreadiidae) and the encapsulation responses. PhD Thesis. 143 p.

Chuchukalo V.I. 2006. [Nutrition and Feeding Relationships between Nekton and Nektobenthos in Far Eastern Seas]. Vladivostok. TINRO-Tsentr. 484 p. [In Russian]

Didenko E.M., Shevshenko G.G. 1999 [Class Acanthocephala] // A.F. Volkov, G.M. Gavrilov, S.E. Pozdnyakov, V.E. Rodin, N.S. Fadeev, V.P. Shuntov, N.S. Samoylova (eds.) [Paraziticheskiye chervi ryb dal'nevostochnykh morey i sopredel'nykh akvatoriy Tikhogo okeana]. Vladivostok: TINRO. P.51-59 [in Russian].

Dubinina M.N. 1971. [Cestodes from fishes of the River Amur basin] // Parazitologicheskii Sbornik. Vol.25. P.77-119 [in Russian].

Dogiel V.A. 1962. General parasitology. Edinburgh and London: Oliver and Boyd. 516 p.

Goedknegt M.A., Feis M.E., Wegner K.M., Luttikhuizen, P.C., Buschbaum C., Camphuysen K., van der Meer J., Thieltges D.W. 2016. Parasites and marine invasions: Ecological and evolutionary perspectives // Journal of Sea Research. Vol.113. P.11-27. doi: 10.1016/j.seares.2015.12.003

Gordeev I.I., Grigorov I.V., Afanasyev P.K. 2017. Infection of the pacific saury Cololabis saira by acanthocephalans in the Kuril Islands area // Parazitologiya. Vol.51. No.1. P.51-56.

Gordeev I.I., Starovoytov A.N., Ponomarev S.S., Shevlyakov A.V., Milovankin P.A. 2018. [Trawl survey of Pacific salmon on the R/V "Professor Kaganovsky" in the northwestern part of the Pacific Ocean (May-July 2018)] // Trudy VNIRO. Vol.171. P.208-213 [in Russian].

Gordeev I.I., Klovach N.V. 2019. [Free Salmon: the Difficulty of Forecasting the Catch of Pacific Salmons] // Priroda. Vol.3. P.22-27 [in Russian]. doi: 10.7868/ S0032874X19030049

Gulyaev V.D., Korotaeva V.D. 1989. [Redescription of the type species of the genus Glossobothrium Yamaguti, 1952 (Cestoda, Pseudophyllidea)] // Voprosy zoologii. Vol.6. P.8-11 [in Russian].

Hudson P.J., Dobson A.P., Lafferty K.D. 2006. Is a healthy ecosystem one that is rich in parasites? // Trends in Ecology \& Evolution. Vol.21. No.7, P.381-385. doi: 10.1016/j.tree.2006.04.007

Iannacone J., Flores L.A. 2013. Parasitological indices of Pacific pomfret Brama japonica Hilgendorf, 1878 (Osteichthyes, Bramidae) acquired at the fishing terminal of Chorrillos Lima, Peru // Neotropical Helminthology. Vol.7. No.1. P.117-132.

Ichihara I., Kato K., Kamegai S., Machida M. 1968. On the parasites of fishes and shell-fishes in Sagami Bay (No. 4) parasitic helminths of Mackerel, Pneumatophorus japonicus (Houttuuyn) // Research Bulletin of the Meguro Parasitological Museum. Vol.2. P.45-60.

Jensen K., Nikolov P., Caira J.N. 2011: A new genus and two new species of Anteroporidae (Cestoda: Lecanicephalidea) from the darkspotted numbfish, Narcine 
maculata (Torpediniformes: Narcinidae), off Malaysian Borneo // Folia Parasitologica. Vol.58. P.95-107. doi: 10.14411/fp.2011.010

Karmanova I.V. 1998. [Parasites of Pacific salmons in the epizootic situation of parasitoses in the Paratunka river basin (Kamchatka)]. PhD Thesis. 24 p. [In Russian]

Klimpel S., Busch M.W., Kellermanns E., Kleinertz S., Palm H.W. 2009. Metazoan deep sea fish parasites. Solingen. Natur \& Wissenschaft. 384 p.

Klimpel S., Palm H.W., Busch M.W., Kellermanns E., Rückert S. 2006. Fish parasites in the Arctic deep-sea: Poor diversity in pelagic fish species vs. heavy parasite load in a demersal fish // Deep Sea Research Part I: Oceanographic Research Papers. Vol.53. No.7. P.1167-1181. doi: 10.1016/j.dsr.2006.05.009

Konovalov S.M. 1971. [Differentiation of local stocks of the red salmon Oncorhynchus nerka (Walbaum).] Leningrad. Nauka. 229 p. [In Russian]

Kovalenko L.M. 1986. [Pacific mackerel acanthocephalans] // Sovremennoye sostoyaniye promysla tuntsov i ekologiya skombroidykh ryb. Tezisy dokladov Vsesoyuznogo soveshchaniya po problemam tuntsovogo promysla 23-25 sentyabrya 1986 g. Kaliningrad. P.154-155 [in Russian].

Kruse G.O.W. 1977. Some digenetic trematodes from fishes of the Bering Sea with the descriptions of Prosorhynchus mizellei sp. n. (Bucephalidae) and Pseudopecoelus nossamani sp. n. (Opecoelidae) // Proceedings of the Helminthological Society of Washington. Vol.44. No.1. P.73-76.

Kuramochi T. 2009. Digenean trematodes of fishes from deep-sea areas off the Pacific Coast of Northern Honshu, Japan // Deep-Sea Fauna and Pollutants off Pacific Coast of Northern Japan. Vol.39. No.39. P.25-37.

Kurochkin Y.V., Pozdnuakov S.E., Kovalenko L.M. 1987. [On the infection of Pacific saury with acanthocephalans and methods of reducing the economic damage caused by them] // Parazity i Bolezni Morskikh Gidrobiontov. VNIRO-PINRO. P.63-75 [in Russian].

Kuznetsova N.A., Shebanova M.A. 2017. [Feeding and trophic relations of mass fish species in the Kuril waters of the Pacific Ocean] // Izvestiya TINRO. Vol.190. P.132-145 [in Russian].

Leigh S.C., Papastamatiou Y., German D.P. 2017. The nutritional physiology of sharks // Reviews in Fish Biology and Fisheries. Vol.27. No.3. P.561-585. doi: 10.1007/s11160-017-9481-2

Love M.S., Moser M. 1976. Parasites of California marine and estuarine fish. Faculty Publications from the Harold W. Manter Laboratory of Parasitology. Paper 749. University of Nebraska, Lincoln. 520 p.

Machida M. 1985. Helminth parasites of cyclopterid fish, Aptocyclus ventricosus, caught off northern Japan // Bulletin of the National Science Museum, Japan, A (Zoology). Vol.11. No.3. P.123-128.

Mamaev Y.L., Parukhin A.M., Baeva O.M., Oshmarin P.G. 1959. [Helminth fauna of far Eastern salmon in relation to the question of local stocks and feeding migrations of these fish.] Vladivostock: DVR. Sib. Otd. AN SSSR, TINRO. 74 p. [In Russian]

Margolis L. 1965. Parasites as an auxiliary source of information about the biology of Pacific salmons (genus Oncorhynchus) // Journal of the Fisheries Board of Canada. Vol.22. No.6. P.1387-1395.

Marti Yu.Yu. 1980. [Migrations of marine fishes.] Moscow: Pishchevaya promyshlennost'. 248 p. [In Russian]

McDonald T.E., Margolis L. 1995. Synopsis of the Parasites of Fishes of Canada: Supplement (1978-1993) // Canadian Special Publication of Fisheries and Aquatic Sciences 122. Nanaimo. 272 p.

Merlo-Serna A.I., Garcia-Prieto L. 2016. A checklist of helminth parasites of Elasmobranchii in Mexico // Zookeys. Vol.563. P.73-128. doi: 10.3897/zookeys.563.6067

Moles A., Heifetz J. 1998. Effects of the brain parasite Myxobolus arcticus on sockeye salmon // Journal of Fish Biology. Vol.52. P.146-151.

Monticelli F.S. 1889. Notes on some Entozoa in the collection of British Museum// Proceedings of the Zoological Society of London. Vol.21-22. P.321-325.

Volkov A.F., Gavrilov G.M., Pozdnyakov S.E., Rodin V.E., Fadeev N.S., Shuntov V.P., Samoylova N.S. (eds.) 1999. [Paraziticheskiye chervi ryb dal'nevostochnykh morey i sopredel'nykh akvatoriy Tikhogo okeana]. Vladivostok: TINRO. 123 p. [In Russian]

Vyalova G.P. 2002. [A variety of parasites chum and pink salmon Sakhalin] // Izvestiya TINRO. Vol.131. P.439459 [in Russian].

Pospehov V.V., Atrashkevich G.I., Orlovskaya O. 2014. [Parasitic worms of migratory salmon of the northern Okhotsk Sea area.] Kordis. Magadan. 129 p. [In Russian]

Quiazon K.M.A. 2015. Updates on aquatic parasites in fisheries: implications to food safety, food security and environmental protection // Journal of Coastal Zone Management. Vol.18. P.396. doi: 10.4172/24733350.1000396

Scholz T., Euzet L., Moravec F. 1998. Taxonomic status of Pelichnibothrium speciosum Monticelli, 1889 (Cestoda: Tetraphyllidea), a mysterious parasite of Alepisaurus ferox Lowe (Teleostei: Alepisauridae) and Prionace glauca (L.) (Euselachii: Carcharinidae) // Systematic Parasitology. Vol.41. P.1-8. doi: 10.1023/ A: 1006091102174

Solovyova G.F. 1999. [Class Nematoda.] // A.F. Volkov, G.M. Gavrilov, S.E. Pozdnyakov, V.E. Rodin, N.S. Fadeev, V.P. Shuntov, N.S. Samoylova (eds.) Paraziticheskiye chervi ryb dal'nevostochnykh morey i sopredel'nykh akvatoriy Tikhogo okeana. Vladivostok: TINRO. P.51-59 [in Russian].

Suyama S., Masuda Y., Yanagimoto T., Chow S. 2019. Genetic and morphological variation in Pennella sp. (Copepoda: Siphonostomatoida) collected from Pacific saury, Cololabis saira // Mar. Biodivers. Vol.49. No.3. P.1233-1245. doi: 10.1007/s12526-018-0901-x

Zmeev G.Y. 1936. [Trematodes and cestodes of the Amur River] // Parazitologicheskii Sbornik. Vol.6. P.305346 [in Russian].

Zschokke F., Heitz F.A. 1914. Endoparasiten aus Salmoniden von Kamtschatka // Revue suisse Zool. Vol.22. No.8. S.195-256.

Responsible editor N.M. Biserova 